\title{
Mechanism of rotatory eye movements in opsoclonus
}

\author{
M. A. GRESTY, L. J. FINDLEY, AND PAULINE WADE \\ From the Medical Research Council, Hearing and Balance Unit, Institue of Neurology, \\ National Hospital, Queen Square, London WC1N 3BG
}

SUMMARY 'Rotatory nystagmus', in which the visual axis of the eye moves involuntarily in the horizontal and vertical planes describing a closed loop trajectory, was analysed by means of combined video and electro-oculography in a patient with multiple sclerosis having numerous ocular signs of cerebellar disease. The rotations were sporadic, isolated single cycles having stereotyped, crescentiform loop shapes. Each consisted of a combination of a single cycle of vertical ocular flutter, the onset of which was followed 40 to 50 milliseconds later by a single cycle of horizontal ocular flutter. The timing relationship between flutters suggest that the vertical and horizontal systems had become somehow entrained. Rotatory nystagmus is saccadic in nature and arises from a unique timing relationship between vertical and horizontal flutter.

Ocular 'flutter' and opsoclonus (or 'saccadomania') are involuntary, abnormal eye movements which can occur with lesions localised to the cerebellar system and when the cerebellum is involved in a more generalised pathological process such as encephalitis. ${ }^{1-3}$ Flutter has the appearance of a rapid to and fro movement of the eye which looks like a rapid tremor. Eye movement recordings show that flutter consists of a sequence of saccades in alternating directions with no intersaccadic latency. Opsoclonus takes the form of multiple saccadic eye movements in random directions, including along the oblique axes.

There is a further involuntary movement occurring in association with the above ocular signs in which the eye appears to rotate quickly as if it were tracking around the perimeter of a disc. This may be referred to as rotatory nystagmus (as opposed to torsional nystagmus, in which the eye rotates about the visual axis) and is usually classified as opsoclonus, although it is not obviously saccadic in nature. We have recorded and analysed rotatory nystagmus in a patient with a predominately cerebellar syndrome and found that the movement consists of a combination of cycles of flutter in the vertical and horizontal axes, with particular phase and velocity characteristics which determine a curved (crescentiform) trajectory.

\section{Case history}

The patient was a 27-year-old male under the care of Professor John Marshall. He had progressive

Correspondence to Dr M. A. Gresty. neurological symptoms without remission for 4 years, commencing with vertigo on upgaze, which had been persistent. Two years prior to recording he developed disabling oscillopsia, and during the last year he had developed progressive cerebellar ataxia of all limbs in association with intermittent paraesthesia in both legs below the knees. There was no significant past family history or past medical history.

On examination of the general systems there were no abnormal findings. He was normotensive. His gait was grossly ataxic. There was obvious intellectual impairment and a dysarthria of cerebellar type. He had persistent titubation of the head at rest. He had a long-standing amblyopic right eye with vision corrected to $6 / 12$ right and 6/6 left. There was a bilateral pendular nystagmus in the horizontal plane which was present in primary gaze and greater in the left eye. Superimposed on the pendular nystagmus were square-wave jerks in both horizontal and vertical directions. Eye movements in the direction of left gaze were dysmetric, and a mild, low-amplitude, left-beating nystagmus replaced the pendular oscillations. There was a mild decentering dysmetria to right gaze together with occasional bursts of macrosaccadic oscillations in the horizontal plane and of flutter in both the horizontal and vertical directions. Pursuit and optokinetic nystagmus were broken during following movements to the left. There were occasional stereotyped rotatory movements in which the globe moved upwards from the midposition and then towards the left, returning downwards and towards the right. These occurred on an average of 1 or 2 per 
Fig. 1 A: An example of flutter-like oscillations in the vertical plane. The flutter consists of 4 cycles of to and fro saccadic movements with no intersaccadic interval. The flutter appeared to be triggered in some way by the initial upwards eye movement. B: An example of rotatory nystagmus. The central Lissajous figure represents the vector sum of movements of the visual axis of the eye in the horizontal $(\mathrm{H})$ and vertical $(\mathrm{V})$ directions. This is what is 'seen' when the eye is examined. Movements in the vertical plane are shown on the left and in the horizontal plane are as shown above. The arrowheads on the Lissajous figure show the direction in which the eye rotated. The arrowheads on the vertical and horizontal eye movement components indicate the synchronisation of the two traces.

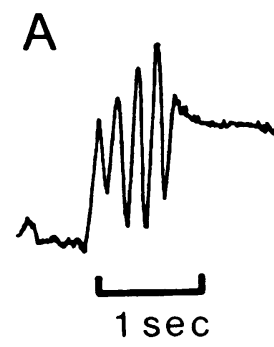

minute. Electro-oculography revealed a mild left internuclear ophthalmoplegia which was not obvious upon examination. There was mild bilateral facial weakness. The lower cranial nerves were normal. All limbs were mildly spastic, but power was normal. The deep tendon reflexes were bilaterally pathologically increased, the abdominal reflexes were absent, and the plantar reflexes were flexor. There was severe ataxia of all limbs, mainly dysmetric in type and evoked particularly on fingernose testing. Rapid repetitive movements of hands and feet were severely impaired.

The investigation results supported the clinical diagnosis of demyelinating disease. The visual evoked potentials (VEPs) in the clinically more normal eye showed clear delay in latencies, the cerebrospinal fluid which was obtained at lumbar puncture was at normal pressure and without cells but with a protein of $105 \mathrm{mg} / 100 \mathrm{ml}(1.05 \mathrm{~g} / \mathrm{l})$ with typical oligoclonal pattern on polyacrylamide-gel electrophoresis. The skull $x$-rays were normal, and the unenhanced CT brain scan was within normal limits.

Conclusions: Multiple sclerosis with predominately cerebellar system involvement, particularly evident in the ocular signs. ${ }^{1}$

ELECTRO-OCULOGRAPHIC ANALYSIS

Recordings of eye movements in the horizontal and vertical planes were obtained by direct coupled electro-oculography. The wave forms were displayed on an electrostatic chart recorder and on an oscillo-

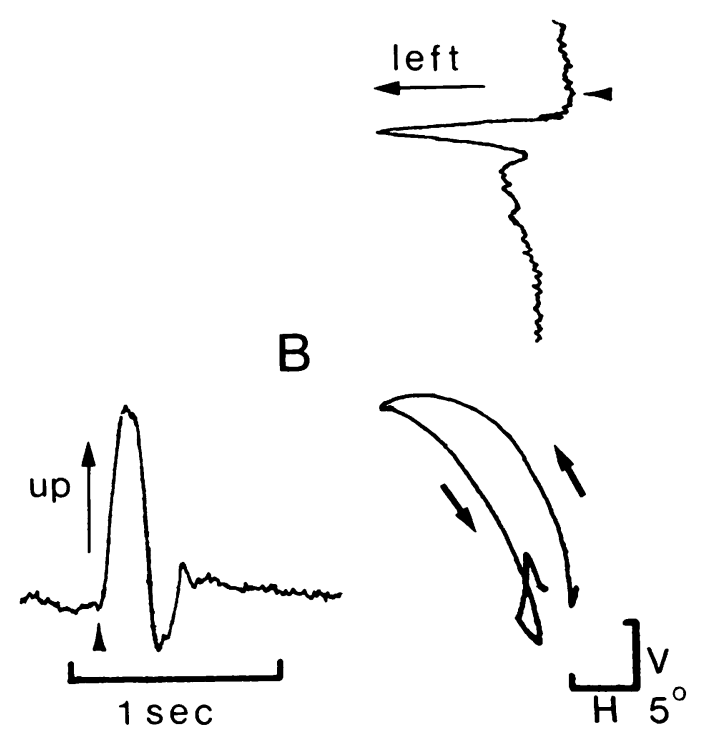

scope screen. The traces on the oscilloscope screen were viewed with a video camera and superimposed on a video picture of the patient's eyes with a video mixer. This enabled us to select samples of rotatory nystagmus and verify that the associated traces were free from artefacts.

A relatively unusual feature of our patient's ocular movements was that he produced flutter eye movements in the vertical plane. These we believe have not been hitherto reported with objective recordings, and a typical example of their occurrence is presented in Fig. 1A. The beats of flutter were always initiated by movement in the vertical direction.

Electro-oculographic recording of the rotatory nystagmus is presented in Fig. 1B. The central Lissajous figure represents the path of rotation of the visual axis of the eye in the transverse plane and is the vector sum of the movements along the horizontal axis, as shown above, and along the vertical axis, as shown on the left. In fact the trajectory followed by the globe during a cycle of rotatory nystagmus was crescentiform and not circular. The vertical and horizontal traces show that the crescentic rotation is composed of synchronised single beats of flutter in the horizontal and vertical directions.

It is only by virtue of certain phase and velocity relationships between the horizontal and vertical flutter that the resultant trajectory of the eye was crescentiform. In the first place the vertical movements always began between $\mathbf{4 0}$ and $\mathbf{5 0}$ milliseconds before the horizontal movement, resulting in the 
movement being predominantly upwards initially and then obliquely as the horizontal movement developed. The net result was that the eye followed the trajectory of the upper curve of the Lissajous figure. Also, there was a delay of about 20 milliseconds between the upwards and downwards saccade, so that despite the latency difference in initiation the downwards and inwards movements were synchronised and of similar velocity, with the result that the eye followed the fairly linear trajectory of the lower curve of the Lissajous figure.

All such eye movements in our patient were of this form. Although they could have been formed by coincidental synchronisation of vertical and horizontal flutter, we formed the strong impression that each occurrence of rotatory nystagmus was a distinct entity, as if the horizontal and vertical systems were coupled such that certain vertical movements would trigger off horizontal ones. We did not record any movements with a more circular trajectory, although there is no prima facie reason why they could not occur.

We have demonstrated that rotatory nystagmus in cerebellar disease consists of synchronised beats of flutter occurring in the horizontal and vertical axes of eye movement. The movement is crescentic in shape because of the timing relationships between the beats of flutter along the 2 axes. This demonstration indicates that rotatory nystagmus should be classified with flutter and opsoclonus.

One curious aspect of rotatory nystagmus is that it is not similar to a normal saccadic movement along an oblique axis, in which case the initiations of the horizontal and vertical components of the movement are virtually simultaneous. On the contrary, the delay of $\mathbf{4 0}$ milliseconds or more between the vertical and horizontal saccades which make up the rotatory nystagmus suggests that an unusual entrainment has developed between the vertical and horizontal systems such that a certain type of vertical movement 'triggers' a stereotyped response in the horizontal plane.

\section{References}

1 Cogan D. Ocular dysmetria; flutter like oscillations of the eyes and opsoclonus. Arch Ophthalmol 1954; 51 : 31835.

2 Hoyt WF, Daroff RB. Supranuclear disorders of ocular control systems in man. In: Bach-y-Rita $\mathbf{P}$, Collins $\mathbf{C}$, eds. The Control of Eye Movements. London: Academic Press, 1971 : 175-235.

3 Daroff RB. Ocular oscillations. Ann Otol Rhinol Laryngol 1977; 86: 102-7. 